\title{
A Comparative Study of Edge Detection Techniques
}

\author{
Ranjeet Kumar Singh \\ Assistant professor Dept. of I.T. \\ SLSET Kichha, (Uttrakhand), India
}

\author{
Ram Bhawan Singh, Ph.D \\ Associate Professor: Dept. of C.S. \\ RBMI, Bareilly, India
}

\author{
Shashank Shekhar \\ Dept of E.C. (Digital Communication)
}

St. Margaret Engineering College, Alwar (Raj.), India

Vinit Chauhan

Assistant Professor: Dept. of ECE

St. Margaret Engineering College, Alwar (Raj.), India

\begin{abstract}
The edge detection is considered as a verysignificant technique in the field of Computer Vision. The term: 'edges' is used to define the boundaries between regions in a particular image, which supports with object segmentation and also in recognition of an object. In this paper authors focus a comparative study of edge detection techniques such as Sobel, Robert, Prewitt and impact of noise on it. The Experiment results find the edges of original image with the help of different type of edge detection technique such as Robert, Sobel and Prewitt and then we notice the quality of image with the help of PSNR, SNR, and Correlation Coefficient.
\end{abstract}

\section{Index Terms}

Edge detection,Noise, PSNR, SNR, Correlation Coefficient.

\section{INTRODUCTION}

Image processing and Computer Vision, edge detection gives the localization of substantialdifferences of a gray image and the documentation of the geometrical and physical characteristic of substances of the scene. The dissimilarities in the gray imagegenerallycontain of discontinuities (step edges), local extreme (line edges) and junctions. Maximum current edge detectors are multi-scale and independentconsist of four main processing Steps: smoothing, Enhancement Detectionand Localization. The main four steps of edge detection are described as follows:

Smoothing: It suppresses as ample noise as possible, without abolishing the true edges.

Enhancement: The use of filter improves the quality of the edges and makes clear object detection (i.e. Sharpening).

Detection: It decides which edge pixels must be rejected as noise and which must be retained (frequently, thresholding offers the standard used for Edge Detection).

Localization: It determines the exact location coordinate of an edge. Edge Thinning and linking operation are required to correctly localize the edge.

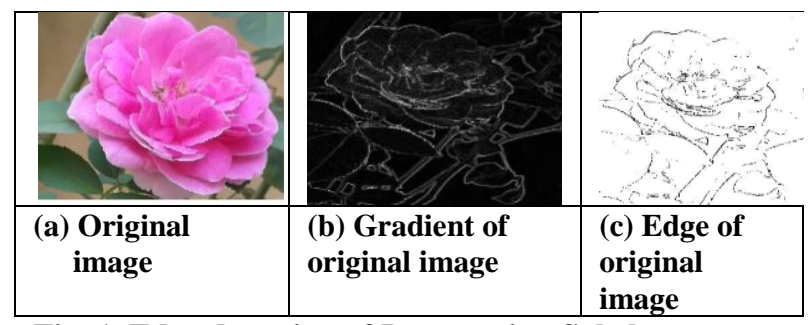

Fig. 1. Edge detection of Image using Sobel operator

\section{EDGE DETECTION TECHNIQUE}

Edge Detection is a technique of finding and identifying the sharp discontinuities presented in an image. The term discontinuities are defined as sudden changes that are made in pixel intensity which describe the boundaries of substances in a scene. All standard methods of edge detection include theConvolving of the image with an operator, and to get big gradients in the image though returning values of zero in constant regions. Mostly, there are numbers of edge detection operators exists, and each of these operations is designed to be sensitive toward the certain types of edges. The programming structure of the operator is applicable for determining the characteristic direction in which direction it is very sensitive to the obtained edges. The operators are developed to look for vertical, horizontal, and diagonal edges. Edge detection is hard to obtain in noisy images, because the noise and the edges are high frequency content [5].

\subsection{Sobel Operator}

For processing of an image the Sobel operator is used,particularly this is used within edge detection algorithms. Technically, Sobel operator is known as discrete differentiation operator. It is used to computing an approximation of the gradient of intensity function for image. The result computed with Sobel Operator considering at each point can be the corresponding gradient vector or vector's norm. It is based on convolving the image by minor, separate, and integer valued filter inverticaland horizontal direction.

[9].The Sobel Operator combination of a pair of convolution of $3 * 3$ metrics as presented in Figure 2.1. One kernel is simply stated on position and the other rotated by $90^{\circ}$.

\begin{tabular}{|l|l|l|}
\hline-1 & 0 & +1 \\
\hline-2 & 0 & +2 \\
\hline-1 & 0 & +1 \\
\hline
\end{tabular}

$\mathrm{G}_{\mathrm{x}}$

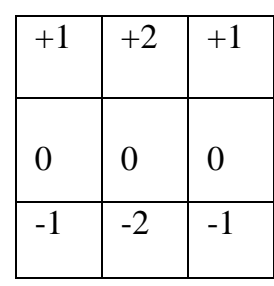

$\mathrm{G}_{\mathrm{y}}$
Fig. 2.1 Masks used by Sobel Operator

The main objective behind designing these kernels are for responding maximally to edges running in vertical and horizontal direction relative to the pixel grid, there is a single kernel is assigned for each of the two perpendicular orientations. For the input image the kernels can be applied separately one by one, for producing the separate 
measurements of the gradient component in various orientations (defined as $G x$ and $G y$ ). It can be joined together to get the estimation of orientation of the gradient. The absolute value of magnitudeof the gradient and the magnitude of gradient is given by [5-8]:

$\operatorname{Mod} G=(G \times 2+G y 2) \frac{1}{2} 2$

Typically, an estimated magnitude of image is computed using:

\section{ModG=ModGx+ModGy}

Which is much faster to compute? In the given below the angle of orientation of edge with object relative to the pixel grid producing values to the spatial gradient.

$\mathrm{q}=\arctan (G y / G x)$

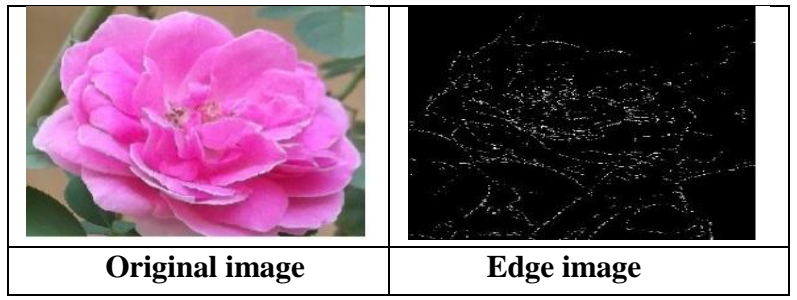

Fig. 2.2 Edge Calculated by Sobel Edge Detection Operator

\subsection{Prewitt's Operator}

Prewitt edge detector operator is identical to the Sobel edge detector operator. Prewitt edge detector operator is used for the detection of horizontal and vertical edges in images [5]. In the below Fig.2.3 it show the Masks for the Prewitt gradient edge detector.

\begin{tabular}{|l|l|l|}
\hline-1 & 0 & +1 \\
\hline-1 & 0 & +1 \\
\hline-1 & 0 & +1 \\
\hline
\end{tabular}

Gx

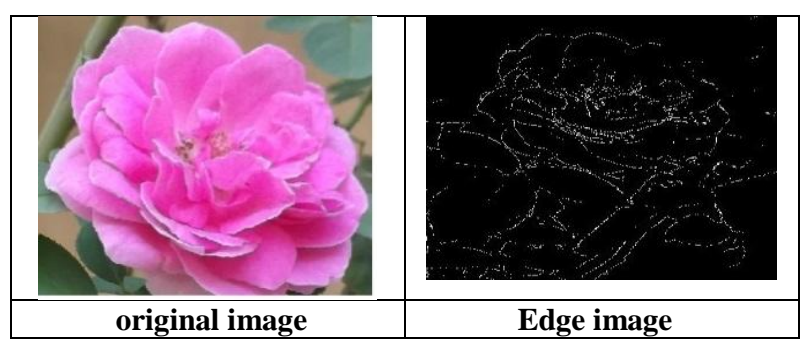

Fig. 2.4 Edge Calculated by Prewitt Edge Detection Operator

\subsection{Robert's Cross Operator}

The Roberts Cross Operator is used to perform a simple, rapid to compute, 2-D spatial gradient measurement on a particular image. Pixel values at every point in the output are used to denote the valued entire magnitude of the spatial gradient of the image that is to be input at that point. The operator contains of a pair of convolution kernels in $2 * 2$ forms as shown in below Figure 2.5. It is same as the Sobel operator because of rotation that occurs in it [5]

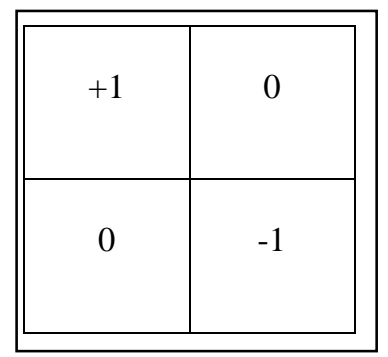

$\mathrm{G}_{\mathrm{x}}$
$\mathrm{G}_{\mathrm{y}}$

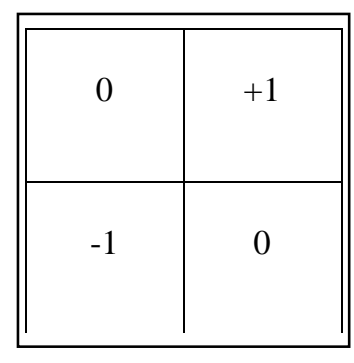

Fig. 2.5 Masks used for Robert operator

These kernels are designed to reply maximal of the edges running on $45^{\circ}$ to the pixel grid where one kernel for both the perpendicular orientations. The kernels can be applied distinctly to the image that is to be input, for producing the distinct measurements of the gradient module in each orientation (call these Gxand Gy). These can then combined together and calculate the complete magnitude of the gradient on each point with orientation of gradient. The Magnitude of gradient is obtained as follows [5]:

\section{$\operatorname{ModG}=(\mathbf{G x 2}+\mathrm{Gy2}) \mathbf{1} / 2(4)$}

The estimated magnitude can be computed using:

\section{ModG=ModGx+ModGy (5)}

The angle at location with the edge gives rise to the spatial gradient,

$\mathrm{q}=\arctan (G y / G x)-3 \mathrm{p} / 4(6)$

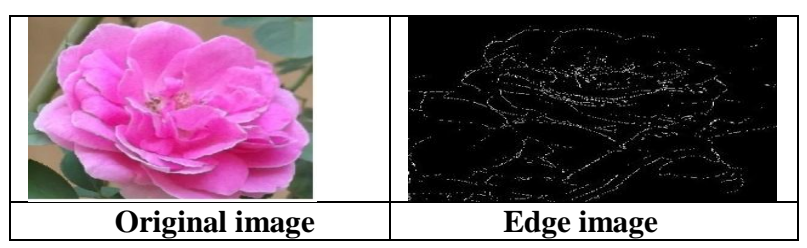

Fig. 2.6 Edge Calculated by Robert Operator

\section{NOISE}

Image noises are the variant of brightness in images formed due to problem with sensor in the digital camera. The image noise sometimes also created in the film grain and in the inevitable shot noise of photon detector [4]. Image Noise is usually regarded as an unwanted by-product of image capture. The unwanted fluctuations in image are also known as "noise". There are several types of noise some important noise are given below:

Amplifier noise (Also known as Gaussian noise)

Salt-and-pepper noise

Shot noise (Also known as Poisson noise)

Speckle noise

\section{EXPERIMENTAL RESULT ANALYSIS}

In The below Figure Author take a Rose Image as an Original Image and Find out the Edge of original image one by one with the help of Sobel, Robert, and Prewitt Edge Detection Operator. In the above experiment is simulated by the MATLAB Software. Here Author measure quality of image which is calculated by Sobel, Robert, and Prewitt operator. In Table 1 its show the quality of edge image in the form of SNR 
(signal to noise ratio), PSNR (Peak signal to noise ratio) and Correlation Coefficient.

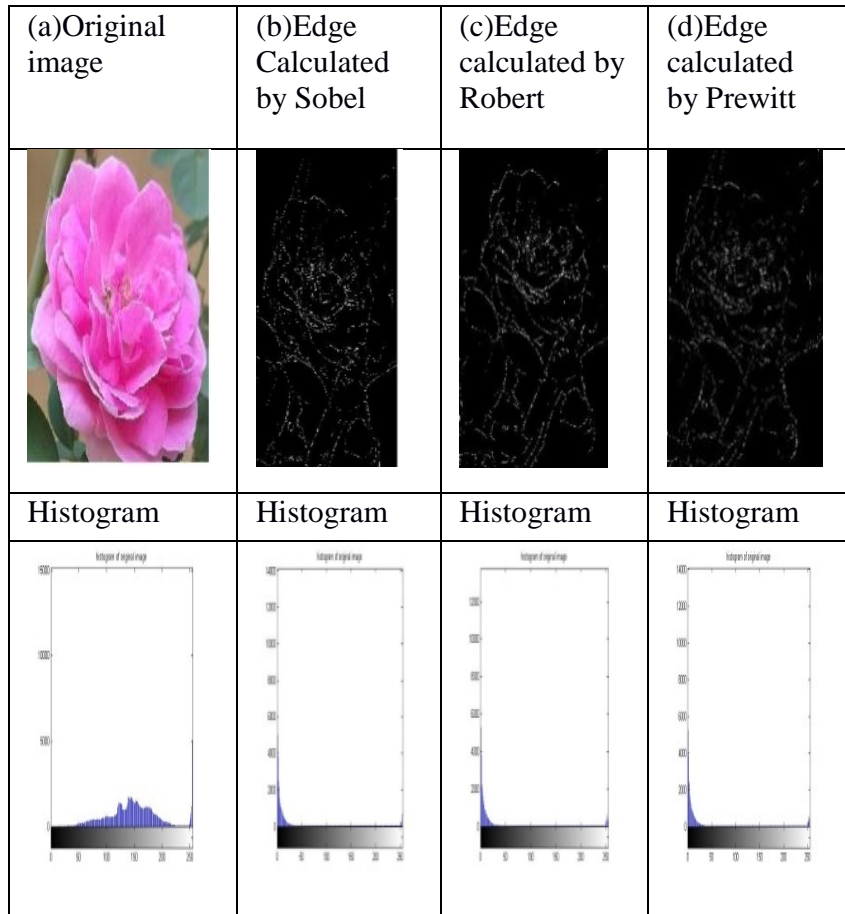

Fig. 2 7 Edge Calculated by Sobel, Robert and Prewitt Edge Detection Operator

In below Table1 show the SNR (signal to noise ratio), PSNR (peak signal to noise ratio) of images in the form of Red, Green and Blue Component.

Table 1: SNR, PSNR, CC comparison between original image and Sobel, Robert, Prewitt edge image with RGB component

\begin{tabular}{|l|l|c|c|c|c|c|c|}
\hline \multicolumn{2}{|l|}{} & \multicolumn{2}{l|}{ SNR } & \multicolumn{2}{l|}{ PSNR } & $\begin{array}{l}\text { Corr. } \\
\text { Coeff. }\end{array}$ \\
\cline { 2 - 7 } & R & G & B & R & G & B & \\
\hline $\begin{array}{l}\text { Original } \\
\text { Image } \\
\text { and } \\
\begin{array}{l}\text { Edge } \\
\text { calculated } \\
\text { by Sobel }\end{array}\end{array}$ & 6.4014 & 9.7326 & 6.9922 & 11.7722 & 15.9275 & 12.6091 & 0.8786 \\
\hline $\begin{array}{l}\text { Original } \\
\text { Image } \\
\text { and } \\
\text { edge } \\
\text { calculated } \\
\text { by } \\
\text { Robert }\end{array}$ & 6.3227 & 9.7626 & 6.9093 & 11.6934 & 15.9575 & 12.5252 & 0.8866 \\
\hline $\begin{array}{l}\text { Original } \\
\text { Image } \\
\text { and } \\
\text { edge } \\
\text { calculated } \\
\text { by } \\
\text { Prewitt }\end{array}$ & 6.2548 & 9.7490 & 6.8426 & 11.6256 & 15.9438 & 12.4595 & 0.8915 \\
\hline
\end{tabular}

Above table focus the SNR values of red component between original image and edge image calculated by Sobel operator is higher than other, and SNR values of green component between original image and edge image calculated by Robert operator is higher compare to other.Similarly we see the
PSNR values of red and blue component between original image and edge image calculated by Sobel operator is Higher Than other and PSNR values of green component between original image and edge image calculated by Robert operator is higher compare to other.

Table 2: SNR, PSNR, CC comparison between original image and Sobel, Robert, Prewitt edge

\begin{tabular}{|l|l|l|l|}
\hline & SNR & PSNR & $\begin{array}{l}\text { Correlation } \\
\text { Coefficient }\end{array}$ \\
\hline $\begin{array}{l}\text { Original Image and } \\
\text { edge calculated } \\
\text { by Sobel }\end{array}$ & 7.7087 & 13.4362 & 0.8786 \\
\hline $\begin{array}{l}\text { Original Image and } \\
\text { Edge calculated } \\
\text { by Robert }\end{array}$ & 7.6648 & 13.3920 & 0.8866 \\
\hline $\begin{array}{l}\text { Original Image and } \\
\text { edge calculated } \\
\text { by Prewitt }\end{array}$ & 7.6154 & 13.3429 & 0.8915 \\
\hline
\end{tabular}

In Table 2 it shows the average SNR and PSNR values or RGB component of images. Finally here we see the SNR values between original imageand edge image calculated by Sobel operator is higher than other, slimily PSNR values between Original Image and edge image calculated by Sobel operator is higher compare to other, and Correlation Coefficient between original image and edge image calculated by Prewitt operator is higher than other. In below we see the output Graph of this experiment.

\section{Output Graph}

The output graph show the experimental result of this experiment which is simulated by MATLAB simulator. In Table 2 it show the SNR between Original image and Sobel, Robert, Prewitt are 7.7087, 7.6648, 7.6154, Similarly PSNR values are $13.4363,13.3920$ and 13.3428. The Correlation Coefficient between them are $0.8786,0.8866,0.8915$.

Coefficient between them is $0.8786,0.8866,0.8915$.

In the above Graph Green Line show the PSNR valuesbetween original image and Edge image calculated by Sobel, Robert, and Prewitt. Similarly Red line Show the SNR values and Black line show the Correlation Coefficient between original image and Edge image calculated by Sobel, Robert, and Prewitt. PSNR stands for peak Signal to noise ratio which forms output in decibels.

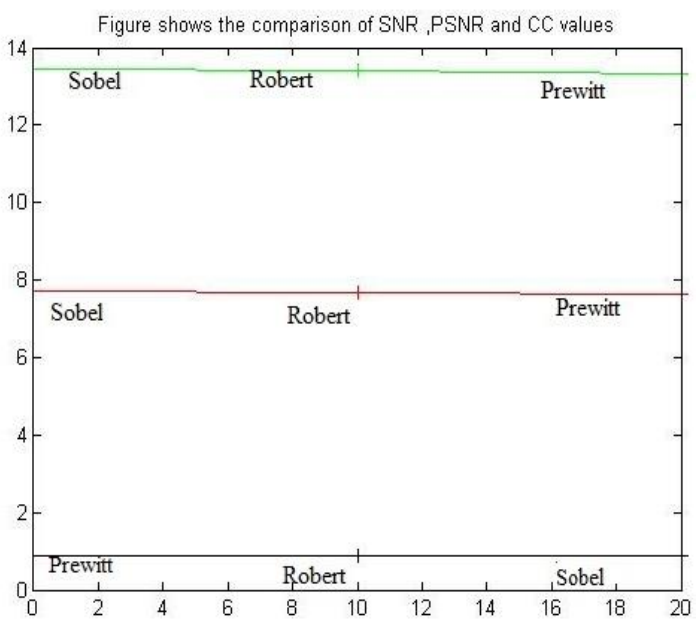


It is used for the purpose of qualitymeasurement of the image or define quality ratio betweenoriginal image and modified image. PSNR values betweentwo images are higher means both image is very near and viceversa. The Correlation Coefficient by two same size images reflects how much similar two image. If the value of correlation coefficient is near to 1 that means both Image is very similar. If value is 1 means both image are same.

Table 3: Comparison of quality of images between EIS, EIR and EIP

\begin{tabular}{|c|c|c|c|c|c|c|c|c|c|c|}
\hline \multirow{2}{*}{$\begin{array}{l}\text { Compa } \\
\text { rison }\end{array}$} & \multicolumn{3}{|c|}{ SNR } & \multicolumn{3}{|c|}{ PSNR } & \multicolumn{3}{|c|}{ MSE } & \multirow{2}{*}{$\begin{array}{l}\mathrm{C} \\
\mathrm{C}\end{array}$} \\
\hline & $\mathbf{R}$ & $\mathbf{G}$ & B & $\mathbf{R}$ & $\mathbf{G}$ & B & $\mathbf{R}$ & $\mathbf{G}$ & B & \\
\hline $\begin{array}{l}{ }_{\text {EIS }} \\
\text { EIR }\end{array}$ & $\begin{array}{l}17 . \\
39\end{array}$ & $\begin{array}{l}17 . \\
39\end{array}$ & $\begin{array}{l}17 . \\
39\end{array}$ & $\begin{array}{l}23 . \\
45\end{array}$ & $\begin{array}{l}23 . \\
45\end{array}$ & $\begin{array}{l}23 . \\
45\end{array}$ & $\begin{array}{l}293 \\
.49\end{array}$ & $\begin{array}{l}293 \\
.49\end{array}$ & $\begin{array}{l}293 \\
.49\end{array}$ & $\begin{array}{l}0.8 \\
99\end{array}$ \\
\hline $\begin{array}{l}\&_{\text {EIR }}^{\text {EIP }} \\
\text { EIP }\end{array}$ & $\begin{array}{l}19 . \\
17\end{array}$ & $\begin{array}{l}19 . \\
17\end{array}$ & $\begin{array}{l}19 . \\
17\end{array}$ & $\begin{array}{l}25 . \\
29\end{array}$ & $\begin{array}{l}25 . \\
29\end{array}$ & $\begin{array}{l}25 . \\
29\end{array}$ & $\begin{array}{l}192 \\
.32\end{array}$ & $\begin{array}{l}192 \\
.32\end{array}$ & $\begin{array}{l}192 \\
.32\end{array}$ & $\begin{array}{l}0.8 \\
94\end{array}$ \\
\hline $\begin{array}{l}\text { EIP } \\
\& \\
\text { EIS }\end{array}$ & $\begin{array}{l}16 . \\
34\end{array}$ & $\begin{array}{l}16 . \\
34\end{array}$ & $\begin{array}{l}16 . \\
34\end{array}$ & $\begin{array}{l}22 . \\
49\end{array}$ & $\begin{array}{l}22 . \\
49\end{array}$ & $\begin{array}{l}22 . \\
49\end{array}$ & $\begin{array}{l}366 \\
.21\end{array}$ & $\begin{array}{l}366 \\
.21\end{array}$ & $\begin{array}{l}366 \\
.21\end{array}$ & $\begin{array}{l}0.8 \\
78\end{array}$ \\
\hline
\end{tabular}

In Table 3, EIS Represent Edge image calculated by Sobel operator, EIR Represent Edge image calculated by Robert operator, and EIP Represent Edge image calculated by Prewitt operator.CC Represent Correlation Coefficient.

\section{CONCLUSIONS}

In this paper author expression Edge DetectionTechnique and it show the brief Introduction of the comparative study of Edge detection operator such as Sobel, Robert and Prewitt. In this experiment Author show the Quality of image (Edge Image) which is calculated by Sobel, Robert, Prewitt etc. The experimental Result is shown in the form of Tabular and Graph

\section{FUTURE SCOPE}

These days, the Digital Watermarking is a biggest field for research combining edge detection on watermarking process makes it bigger and vaster.Infuture, I would like to extend this research in following way.

- To develop an application who remove the impact of noise of edge of image using different type of filter.

- To use Edge Detection technique in digital image watermarking for the purpose of highly authentication of cover image

\section{REFRENCES}

[1] Baisa L. Gunjal, R.R. Manthalkar" an overview oftransform domain robust digital image watermarking algorithms" Journal of Emerging Trends in Computing and Information Sciences, ISSN 2079-8407 Volume 2 No. 1
[2] Abdullah Bamatraf, Rosziati Ibrahim and Mohd.NajibMohd. Salleh" A New Digital Watermarking Algorithm Using Combination of Least Significant Bit (LSB) andInverse Bit" journal of computing, volume 3, issue 4, April 2011, ISSN 2151-9617

[3] SOBEL, I., An Isotropic $3 \times 3$ Gradient Operator, Machine Vision for Three -Dimensional Scenes, Freeman, H., Academic Pres., NY, 376-379, 1990.

[4] SOBEL, I., Camera Models and Perception, Ph.D. thesis, Stanford University, Stanford, CA, 1970.

[5] PREWITT, J., Object Enhancement and Extraction, Picture Processing andPsychopictorics (B. Lipkin and A.Rosenfeld, Ed.), NY, Academic Pres., 1970.

[6] ROBERTS, L. G., Machine Perception of ThreeDimensional Solids, in optical and ElectroOpticalInformation Processing ( J. Tippett, Ed.), 159197, MIT Pres., 1965.

[7] CANNY, J., A Computational Approach to Edge Detection, IEEE Transactions on Pattern Analysis and Machine Intelligence, 8, 679-700, 1986

[8] ZIOU, D. and TABBONE, S., Edge Detection Techniques - An Overview, Technical Report, No. 195, Dept. Math \&Informatique, University de Sherbrook, 1997.

[9] SHIGERU, A., Consistent Gradient Operators, IEEE Transactions on Pattern Analysis and Machine Intelligence, 22 (3), 2000.

[10] Xiang Gen Xia, Charles Boncelet, and Gonzalo Arce (1998) Optics Express, 3(12), 497-511.

[11] Sobel operator - Wikipedia, the free encyclopedia"en.wikipedia.org/wiki/Sobel_operator\#Sim plified description.

[12] SOBEL, I., An Isotropic $3 \times 3$ Gradient Operator, Machine Vision for Three -Dimensional Scenes, Freeman, H., Academic Pres., NY, 376-379, 1990.

[13] SOBEL, I., Camera Models and Perception, Ph.D. thesis, Stanford University, Stanford, CA, 1970

[14] PREWITT, J., Object Enhancement and Extraction, Picture Processing and Psychopictorics (B. Lipkin and A.Rosenfeld, Ed.), NY, Academic Pres., 1970.

[15] ROBERTS, L. G., Machine Perception of ThreeDimensional Solids, in optical and Electro-Optical Information Processing ( J. Tippett, Ed.), 159-197, MIT Pres. 1965.

[16] CANNY, J., A Computational Approach to Edge Detection, IEEE Transactions on Pattern Analysis and Machine Intelligence, 8, 679-700, 1986

[17] ZIOU, D. and TABBONE, S., Edge Detection Techniques - An Overview, Technical Report, No. 195, Dept. Math \&Informatique, University de Sherbrook, 1997. 